\title{
A New Approach to Region Based Image Retrieval using Shape Adaptive Discrete Wavelet Transform
}

\author{
Lakhdar BELHALLOUCHE ${ }^{1}$ \\ ${ }^{1}$ Department of Electronics, Djillali Liabes University, Sidi Bel-Abbes, Algeria \\ E-mail: lakhdar_belhallouche@ @otmail.fr \\ Kamel BELLOULATA ${ }^{1}$, Kidiyo KPALMA ${ }^{2}$ \\ ${ }^{1}$ Department of Electronics, Djillali Liabes University, Sidi Bel-Abbes, Algeria \\ ${ }^{2}$ UEB INSA IETR Département Image et Automatique, 35708 Rennes, France \\ E-mail: Kamel.Belloulata@USherbrooke.ca, kidiyo.kpalma@insa-rennes.fr
}

\begin{abstract}
In this paper, we present an efficient regionbased image retrieval method, which uses multi-features color, texture and edge descriptors. In contrast to recent image retrieval methods, which use discrete wavelet transform (DWT), we propose using shape adaptive discrete wavelet transform (SA-DWT). The advantage of this method is that the number of coefficients after transformation is identical to the number of pixels in the original region. Since image data is often stored in compressed formats: JPEG 2000, MPEG 4...; constructing image histograms directly in the compressed domain, allows accelerating the retrieval operation time, and reducing computing complexities. Moreover, SADWT represents the best way to exploit the coefficients characteristics, and properties such as the correlation. Characterizing image regions without any conversion or modification is first addressed. Using edge descriptor to complement image region characterizing is then introduced. Experimental results show that the proposed method outperforms content based image retrieval methods and recent region based image retrieval methods.
\end{abstract}

Index Terms-Content-based image retrieval (CBIR), DWT, Region-based image retrieval (RBIR), SA-DWT.

\section{INTRODUCTION}

Nowadays, many image-based applications require quick and efficient research algorithms for image databases managing and access. For this purpose, many approaches have been proposed in image indexing and retrieval research field. Traditionally, the most common CBIR methods exploit global image attributes such as color, texture or shape for image characterization [2]. In other words, CBIR method is to firstly construct the image index from extracted features, and then compare resulting index to database images index. Only candidates images with small differences are returned, as similar candidate images to the query one $[1,3]$. The problem with these methods is that they consider image as homogeneous entity. Moreover they can't capture local characteristics of image regions, because they are based on global features.

The region-based image retrieval approach (RBIR) makes it possible to overcome this drawback, by characterizing separately each image region based on local features. These homogeneous image regions are resulted from image segmentation step. Each characterized image region is then matched with all candidate image regions [1, 4]. Similarly to CBIR techniques the RBIR methods can be classified into two major categories: 1) RBIR methods in pixel domain and 2) RBIR methods in the transformed domain. Methods in the first category commonly use the pixels features such as color, texture to characterize each region of the image. These methods are robust to background complexities, occlusion and object distortion. For this reason, several methods have adopted this retrieval approach. Tsai et al., for example, have proposed to segment the image into 5 regions (the upper-left, upper-right, lower-left, lowerright and center regions), and then characterize each region separately by color and texture histograms [5]. Similarly Viminal et al in [6] propose dividing the image into nine sub regions; then each region is represented by a color histogram. Exhaustive review of color and texture based RBIR methods is presented in [4]. The problem with these methods is that, they don't characterize the color distribution in the space domain. A Recently proposed solution, use mid-level features based methods: In [7] for example, Lowe et al propose using scaling invariant feature transform (SIFT). This allows space description notion, by considering image intensities around interesting point's locations. The detection of the interest points is based on scale-space extrema of differences of Gaussians (DOG) within a difference of Gaussian pyramid; the extracted features are then clustered and organized into a set of vectors as image index [7, 8]. This method is then improved in [8] by integrating the local binary pattern (LBP) descriptor, to describe texture feature. In other words the proposed 
histogram in this work is the concatenation of image SIFT descriptor and LBP descriptor.

The state of the art of RBIR methods shows that methods in the second category provide more information about texture and color variations in both the spatial and spectral domains [4]. Discrete wavelet transform for instance can capture local spectral features at multiple resolutions. It has an important energy compaction and coefficient decorrelation properties. Also it is known that DWT is very fast computing transformation tool [9], hence most RBIR systems are based on wavelet and related wavelet coefficients. However, image retrieval approaches based on DWT, are not sensitive to edge singularities [10, 11, 12]. For this reason two alternatives are provided, one is using Gabor filters [13], and the other is using Curvelet transform [9]. In contrast to wavelet transform, Gabor filters can capture texture features at multiple orientations and multiple scales [9]; this is produced in significant improvements of image retrieval results [12, 13, and 14].

On the other hand, curvelet transforms make it possible adapting transform to curved edges regions [9]. Therefore curvelet transform captures efficiently edge features at different orientations and different scales [9]. The aim of this paper is to propose a new method to: firstly overcome edge feature extraction by DWT. And finally, to reduce the gap between low level features, and high semantic object in the scene. In the following, a review of related work is given. Our proposed work is presented in section III. In section IV; we compare our region based image retrieval method with other CBIR and RBIR methods, based on ARR, Precision and Recall parameters. In the last section we present our prospect work and then conclude the paper.

\section{RELATED WORK}

In [4] authors present several region based image retrieval schemes using DWT. Characterizing irregular image regions still remains a difficult problem to image retrieval. Approaches proposed in [15, 16, and 17] appear to be relatively adapted to irregular regions characterization, without data change or modification. The idea is to apply DWT on the image. Region coefficients are then clustered into homogeneous groups. Each DWT coefficients group corresponds to an image region. Nevertheless, obtained results remain poor because of complex coefficients clustering operation: as shown in $[15,16]$, coefficients of the same image region can be classified into different coefficients clusters.

To avoid this regions clustering difficulty, image regions must be segmented firstly and then the resulting local clusters can be grouped if they are significantly similar. In [17], Sun at al have adopted this method. Regions are located by using sliding windows of varying sizes. A dynamic wavelet transformation is applied on each image region. Finally, energy of wavelet coefficients is used to construct the region feature vector. This method provides to address wavelet problems, such lack of scale, translation, and orientation-invariance. In contrast to this method, Zhang et al in [9] apply curvelet transform on modified image regions. In fact, they propose converting each irregular region form into a regular region form, after applying curvelet transform. To permit a texture feature extraction from arbitraryshaped regions in RBIR system, Liu et al $[36,37]$ used an iterative approach based on the theory of successive Projections On to Convex Sets (POCS) which they borrowed from. POCS algorithm is the iterative technique and the iterative process is terminated when the pixels outside the boundary converge. The results are compared with zero padding [36, 37]. This way can results in significant change of image information. All these methods have been proven to be very powerful solutions to detect and describe local features in images, and provide more control on image clustering operation. However, as is demonstrated in [20, 21] these methods are not able to represent efficiently regions semantics. Extrapolation-based methods (Padding methods) aim at extending arbitrarily shaped image segments to a regular block grid, such that common transform techniques (DCT, DWT) can be applied. A simple extrapolation technique is to pad out the segment of interest with a constant intensity, which leads to discontinuities at the border between original and padded image data. Applying the DCT/DWT to these discontinuities, results in a considerable number of nonzero high-frequency coefficients; and a low energy concentration. Also, these approaches involve both spatial- and frequency-domain operations for signal extrapolation (POCS), which is not desirable from computational point of view. Usually, such method does not perform well since fixed-value padding, results in sharp intensity transitions at the region boundary that produce high frequency in the DCT/DWT domain. Unlike extrapolation techniques, Shape-Adaptive methods process pixels inside the segment of interest only; pixels out of the segment remain undetermined, as they do not impact any operation. This class of methods limits the basis functions of a transform to region's shape; shapeadaptive transforms result. For the coding of arbitrarily shaped image segments, it has been reported that shapeadaptive methods outperform extrapolation methods in terms of rate-distortion characteristics.

\section{PROPOSED METHOD}

In this work, a new region-based image retrieval method is introduced. It is novel in the following ways:

- By segmentation of the image into homogeneous regions, before image wavelet transformation, each region is then transformed with SA-DWT independently.

- This method allows to maintain spatial correlation among the different sub-bands of the wavelet transform, and to capture local description of the color and texture features.

Taking advantage of the combined texture and color 
description proposed in [19], the proposed method uses the high wavelet sub-bands coefficients to describe the texture feature, and approximation wavelet sub-band coefficients to describe the color feature.

As mentioned in [22] the drawback of the wavelet transform is that it can describe features only in horizontal, vertical and diagonal directions, and it does not capture the edge information. Unlike the method described in [9] which proposes using the curvelet transform combined to POCS extrapolation technique, to overcome this drawback, in our approach we propose to complete the texture and color region descriptor with a wavelet based directional local extrema patterns (DLEP) description. Directional local extrema patterns was introduced by Subrahmanyam $\mathrm{M}$ as extraction texture method in $0^{\circ}, 45^{\circ}, 90^{\circ}$ and $135^{\circ}$ bloc orientations [22]. The proposed DLEP algorithm, allows extracting directional edge information based on local extrema. In our case we propose to apply this algorithm on low wavelet sub band coefficients. This results in $0^{\circ}, 45^{\circ}, 90^{\circ}$ and $135^{\circ}$ directions description.

Since the great amount of image is stored in JPEG, MPEG or JPEG 2000 formats, the research on image retrieval approaches in the compressed domain; attracts significant attention. This allows reducing time consuming during the retrieval. For this reason, the proposed algorithm is based on image characterization directly in the transformed domain, to avoid time consuming during the inverse transformation. The improvement provided by SA-DWT is due to its ability to work on each image region separately, and preserve its spatial and spectral properties, even the correlation across wavelet sub bands is preserved; in added, just a few wavelet coefficients are used to store the wavelet energy. On the other hand, we believe that combining the color and texture features with the texture orientations features captured by DLEP algorithm, provides a best textured region characterization. Global framework of the proposed technique is presented in Fig.2. As illustrated in this framework, the proposed technique includes the principal axis:

- Images pre-processing: background $\left(\mathrm{r}_{1}\right)$, foreground $\left(\mathrm{r}_{2}\right)$ regions extraction. RGB image conversion into $\mathrm{Y}, \mathrm{C}_{\mathrm{b}}, \mathrm{C}_{\mathrm{r}}$ components.

- Applying SA-DWT on $r_{1}, r_{2}$ image regions.

- Image regions histograms construction.

- Image matching.

\section{A. Regions transformation based on SA-DWT}

In this section we briefly explain discrete wavelet transform method, and how it is used in SA-DWT method.

\section{Discrete wavelet transform}

In 1882 Joseph FOURIER has proposed new signal analysis tool: the Fourier Transform. The idea is to project the one or two dimensions signal on frequency basis functions. Given for example $\mathrm{f}(\mathrm{t})$ as image function.
Discrete Fourier transform off is obtained by:

$$
f(\mathrm{k}, \mathrm{l})=\frac{1}{\mathrm{M}} \frac{1}{\mathrm{~N}} \sum_{\mathrm{m}=0}^{\mathrm{M}-1} \sum_{\mathrm{n}=0}^{\mathrm{N}-1} \mathrm{f}(\mathrm{m}, \mathrm{n}) \mathrm{e}^{-2 \mathrm{i} \pi \mathrm{k} \frac{\mathrm{m}}{\mathrm{M}} \mathrm{e}^{-2 \mathrm{i} \pi \mathrm{l} \frac{\mathrm{n}}{\mathrm{N}}}}
$$

Where:

$k, l$ represent horizontal, vertical frequency coordinates. $m, n$ indicate horizontal, vertical pixel coordinates respectively.

$M, N$ represent image size.

Fourier transform has been used to extract efficiently frequency information. However, any spatial or temporal information cannot be obtained by this mathematical transformation. To overcome this drawback, Gabor has proposed Fourier transform with translating window [23] This solution allows having a better spatial resolution than the Fourier transform, however this technique assumes the used window has a fixed size, and cannot be adapted to all frequency variations in the signal. To address this problem, searchers have developed a new signal analyzing tool based on mathematical functions called the wavelet functions [23]. This transformation provides spectral and space localization. The initial signal is projected on a set of basis functions which vary in frequency and space. In other words, these basis functions are adapting to the frequencies of the signal to be analyzed. Thus, wavelet transformation is located in spectral/time space [24]. The wavelet transform of one dimension signal $S(\mathrm{t})$ is computed by:

$$
S(a, b)=\frac{1}{\sqrt{\mathrm{a}}} \int_{-\infty}^{+\infty} \mathrm{S}(\mathrm{t}) \psi_{\mathrm{a}, \mathrm{b}}^{*}\left(\frac{\mathrm{t}-\mathrm{b}}{\mathrm{a}}\right) \mathrm{dt}
$$

$\psi$ represents the wavelet mother function.

a, b represent scaling and translating coefficients respectively.

All wavelet functions $\psi_{\mathrm{a}, \mathrm{b}}(\mathrm{t})$ are generated by scaling and translating of the wavelet mother function $\psi(\mathrm{t})$. To reduce wavelet complexities, Mallat and Meyer have proposed in [25] a multi resolution signal analysis tool.

Applying DWT on image can be achieved in each image direction independently. First each image row is transformed with 1D DWT; the resulted columns are then transformed by the same wavelet transform. The wavelet transform extension to $2 \mathrm{D}$ space leads to one approximation wavelet sub band LL and 3 details wavelet sub bands (HL, LH, HH), an example of image decomposition by wavelet transform is given in Fig.1.
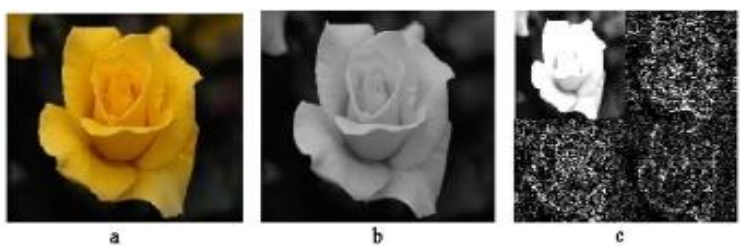

Fig.1. Illustration of an Example of Image Transformation Using DWT: a: Original Image, b: Original Image in Gray Level Space, c: Result Sub Bands Wavelet Transform.

In the image characterizing field, approximation 
wavelet wavelet sub bands can be used to characterize color, shape, and edge.... While the high wavelet sub bands can describe texture feature in horizontal, vertical and diagonal directions. However, this method of features characterization isn't robust to region translation, rotation and scaling change. To solve this problem, we transform each region independently from other image regions, by applying shape adaptive discrete wavelet transform.

\section{Shape-adaptive discrete wavelet transform (SA-DWT)}

In their paper [20], Li et al. have proposed a new discrete wavelet transform for arbitrarily shaped object. The idea was to firstly perform a 1D DWT decomposition on region rows, and then apply the same DWT on resulting region colomns. In order to overcome the drawbacks of the region boundaries (leading and trailing), the boundaries must be extended.

This resulting in pixels values outside the segment related with the pixels values inside the segment. Since we use an odd biorthogonal wavelet filters, the segment extension used is the symmetrical one. The proposed method is based on image mask information. In other words, only image texture containing in the region of interest (ROI) is transformed. The image mask may be defined as image texture within the edge.

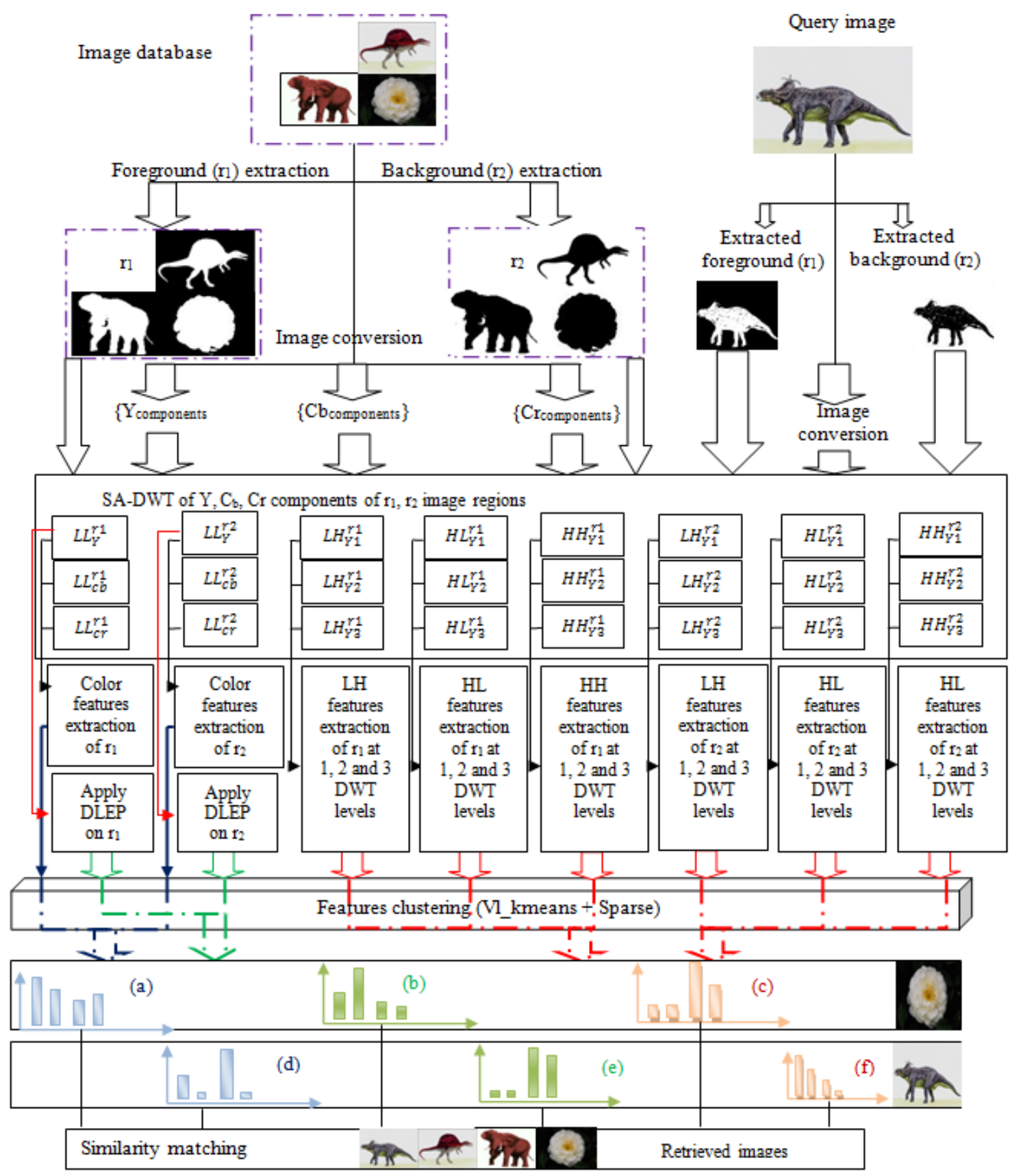

Fig.2. Proposed Framework for RBIR Based on SA-DWT. Query and Images in the Database are Segmented into Foreground and Background Regions. SA-DWT use Image Components and Regions Masks to Compute SA-DWT Coefficients. (a), (b), (c): Color, Edge, Texture Histograms Respectively of Candidate Image. (d), (e), (f): Color, Edge, Texture Histograms Respectively of Query Image. 


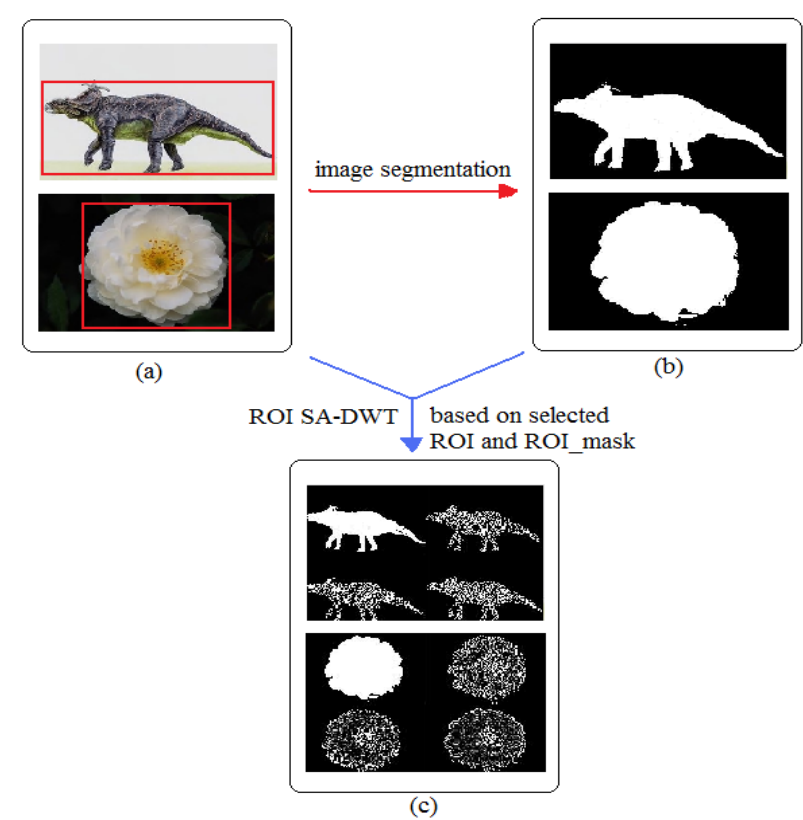

Fig.3. Illustration of Applying SA-DWT on 2D Image. In This Example, Objects Within the Red Box in (a) Are Considered as ROI, Which Should Be Transformed by SA-DWT, (b) Contains Regions Obtained after Image Segmentation, (c) Shows ROI Decomposition with the SA-DWT. Only ROI Is Transformed.

The mask permits ROI pixels to be distinguished from their surroundings. As we can observe on Fig.3, SADWT allows transforming only the foreground of image, considered in this example as the ROI. Rather than using extrapolation or shifting of image pixels, SA-DWT allows preserving rows, and columns positions, in each wavelet transform level.

In algorithm 1, we present SA-DWT algorithm.

Algorithm 1:

Inputs: RGB image I, image mask $S$, wavelet decomposition level $=k$, low pass filter lp, high pass filter $h p$.

Step 1: Convert the RGB image into gray level image.

Step 2: Use $S$ Locate the first segment in the first row of the ROI.

Step 3: Apply 1D-DWT on the located segment in the horizontal direction.

Step 4: Repeat Step 2, 3, for all segments rows.

Step 5: Return the horizontal-transformed version of the ROI.

Step 6: Use $S$ to locate the first segment in the first column of the transformed ROI.

Step 7: Apply 1D-DWT on the located segment.

Step 8: Repeat Step 6, 7, for all segments columns.

Step 9: Return the transformed ROI.

Outputs: Transformed ROI.

In algorithm 1, rows and columns segments can be odd or even length. Applying 1D-DWT on segment $R$ is to perform the following operations:

- Segment extension with symmetrical extension.

- Segment convolution with low and high pass filter:
Convolution low-pass filter:

$$
R_{\mathrm{H}}^{*}(\mathrm{u})=\sum_{\mathrm{v}=0}^{\mathrm{L} / 2} l_{\mathrm{p}}(\mathrm{u}+\mathrm{v}-\mathrm{L} / 2+1) R(\mathrm{~L}-1-\mathrm{v})
$$

Convolution with high-pass filter:

$$
R_{\mathrm{V}}{ }^{*}(\mathrm{u})=\sum_{\mathrm{v}=0}^{L / 2} h_{p}(\mathrm{u}+\mathrm{v}-\mathrm{L} / 2+1) R(\mathrm{~L}-1-\mathrm{v})
$$

$R_{\mathrm{H}}{ }^{*}, R_{\mathrm{V}}{ }^{*}$ represent segment convolution in horizontal and vertical directions respectively.

- Sub-sampling of $R_{\mathrm{H}}{ }^{*}, R_{\mathrm{V}}{ }^{*}$. Therefore, we obtain N/2 coefficients in low-pass sub band, and N/2 in highpass sub band.

\section{B. Region based image retrieval system}

In this section, we formally detail the description of the texture-, color- and the edge based image retrieval method. Commonly, the image description is to construct a set of histograms from local or global features. Experimental results in [19], show that the proposed method in [19] outperforms existing DWT based CBIR methods (GCG: Gaussian Copula with Gamma distributed margins, GFP: Gaussian distribution with Fixed Point covariance matrix estimators). For this reason a part of our indexing scheme is based on the method proposed in [19]. On the other hand, we propose to construct edge histograms according to DLEP algorithm. In the proposed work, DLEP is applied on low wavelet sub band. This part of the paper is reserved exclusively to represent the algorithm of color, texture, and edge histograms construction.

\section{Texture histogram construction}

It is known that $\mathrm{Y}$ component of an image can easily represent the image gray level [19]. For this reason, in their paper Bai et al. have proposed characterizing the texture image features, by high level sub bands wavelet coefficients of the $\mathrm{Y}$ component. In this method, the input segments lengths assumed to be even. However, the SA-DWT deals also with odd segment length. So, for each odd segment length, we propose padding resulting high sub band wavelet coefficients with zero values. This allows getting the same segment length in all high sub bands wavelet coefficients.

We use the Vl-Kmeans algorithm [26] to cluster database vectors to a set of centroid groups. The resulting vectors are then used to generate the image histograms using the sparse representation [27]. An example of texture features construction is given on Fig.4.

As illustrated in this figure, we pad some texture vectors with zero elements. This results in similar number of wavelet coefficients in wavelet sub bands. Each texture vector is constructed form wavelet coefficients. These coefficients are extracted from the same location, in LH, HL, HH wavelet sub bands. More details about this method are in algorithm 2. 
Algorithm 2:

Input: wavelet sub bands $W$ (high frequency sub bands wavelet transform with level decomposition $=3$ ), resulted from algorithm 1 .

Step 1: Construct texture vectors.

Each vector contains 3 wavelet coefficients, which are extracted from the same location. If there isn't enough wavelet coefficients (less than 3 coefficients), we must pad texture vector with zeros elements.

Step 2: For each image class, we group all texture vectors in referred histogram $\left(\mathrm{His}_{\mathrm{ref}}\right)$. Vl_kmeans [26] is then applied to cluster vector groups. Resulted vectors are organized in $\left(\mathrm{His}_{\text {center }}\right)$ histogram. In other words, at the end of this step, we have three His centroid $_{\text {. }}$ histograms for $\mathrm{LH}, \mathrm{HL}$ and $\mathrm{HH}$ sub bands.

Step 3: Construction of texture histograms with sparse algorithm. Here, we don't explain the sparse algorithm; more details are presented in [27].

Output: His LH $_{\text {His }}$ HL $_{\text {His }}$ histograms.
Texture histograms in Fig.5 are the concatenation of texture histograms in horizontal, diagonal and vertical directions. Similar images (elephant images) have produced similar foreground histograms $(a, b)$, histogram (c) is clearly different. However, we can observe that all images in this example have similar background region (the sky). For this reason histograms d, e, f show that there is no practical difference between background regions. The solution is to describe color levels distribution of the sky region.

\section{Color histogram construction}

Color characterization is to combine each 3 low sub bands wavelet coefficients $\left(L L_{Y}, L L_{C b}, L L_{C r}\right)$. These coefficients are from the same location.
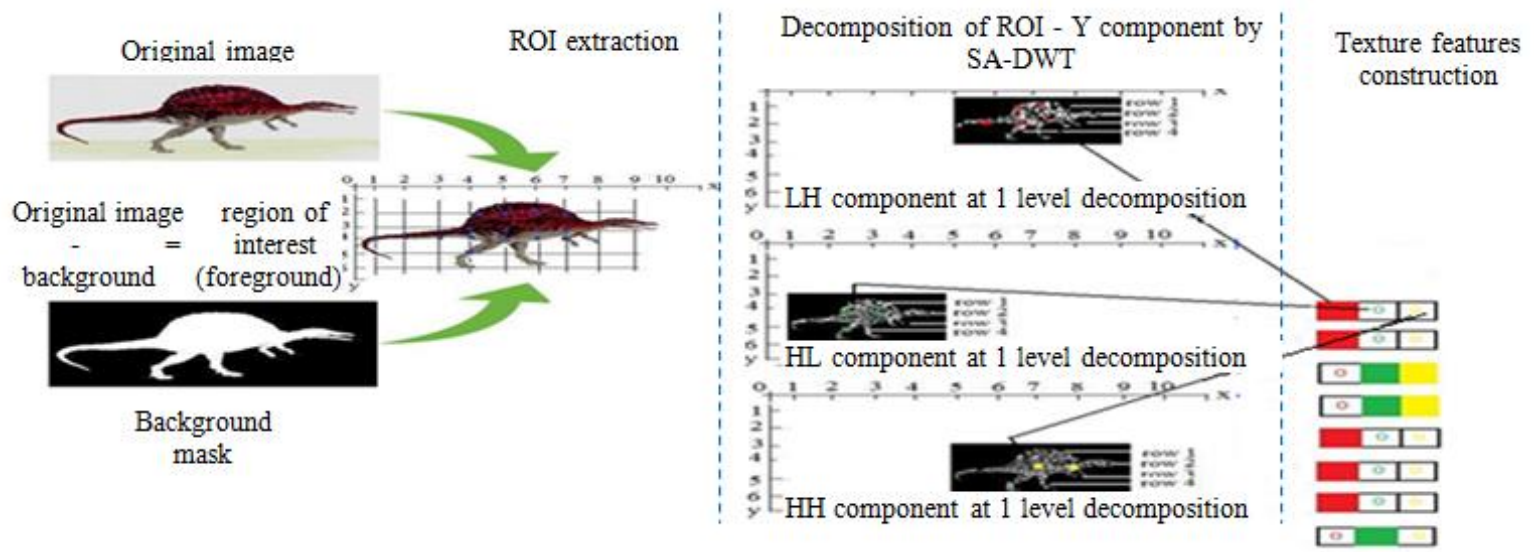

Fig.4. Illustration of Texture Features Construction Method using SA-DWT, in the ROI Extraction, Bleu Points on the Selected Region Represent an Arbitrary Chosen Set of Region Pixels. In the Decomposition Part, Only Y Component of the ROI is Transformed, Red, Green, Yellow Points on the Sub Bands Represent Wavelet Coefficients. In the Texture Features Construction Part, We Represent Only Construction of one Feature, Similarly Remain Features Are Constructed.

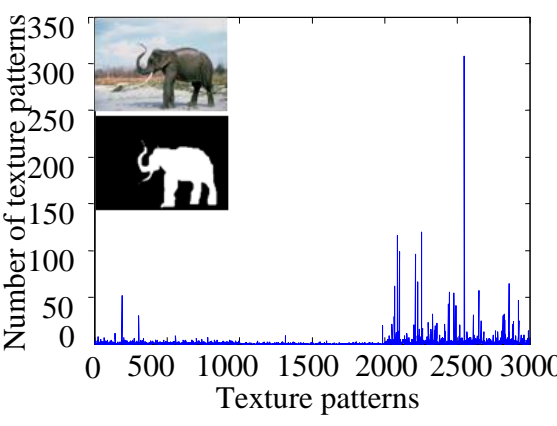

(a)

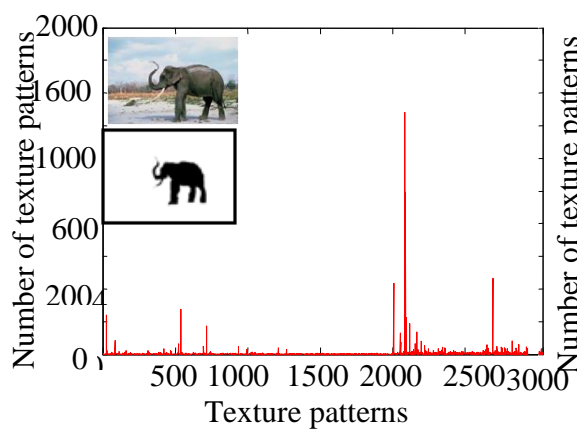

(d)

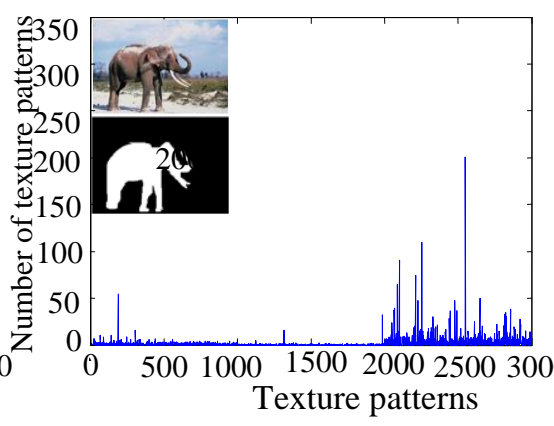

(b)

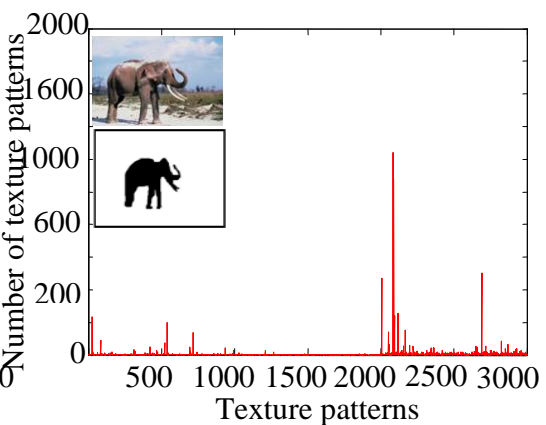

(e)

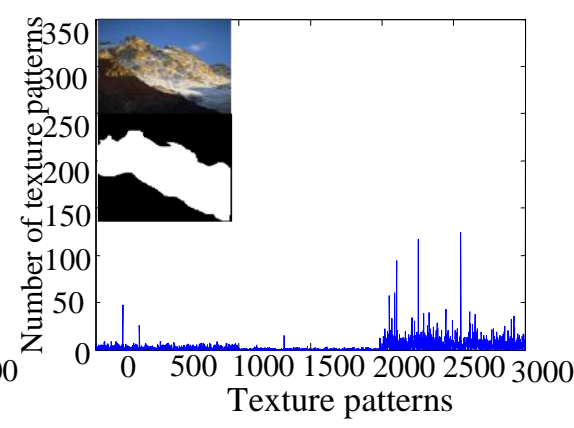

(c)

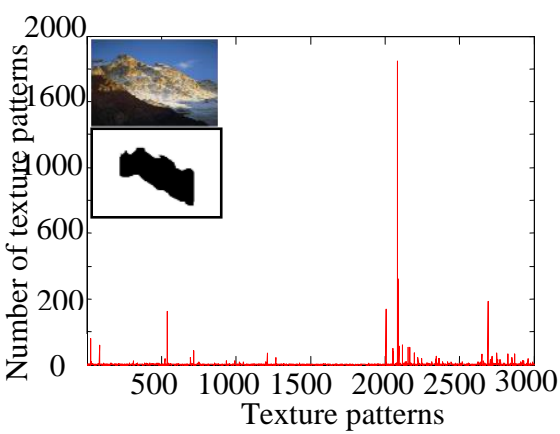

(f)

Fig.5 Some Examples of Texture Histograms. (a), (b), (c) Texture Histograms of Foreground Regions of two Elephant Images and one Mountain Image. (d), (e), (f) Texture Histograms of Background Regions. 
As is shown in Fig.6, here we don't need padding. Algorithm 3 explains color histogram construction from color features. The combined texture histogram contains 3000 bins (1000 bins from each wavelet sub band pattern).

Algorithm 3:

Input: wavelet sub bands $W$ (low frequency sub bands wavelet transform with level decomposition $=3$ ), resulted from algorithm 1 .

Step 1:Construct color vectors as is shown in Fig.6.

Step 2: For each image class, we group all color vectors in referred histogram $\left(\right.$ His $\left._{\text {ref }}\right)$. Color vectors are grouped in one centroid histogram $\left(\mathrm{His}_{\mathrm{center}}\right)$ for each class, with Vl_kmeans [26]. Step 3: Construction of color histograms with sparse algorithm.

Output: His Col $_{\text {histograms. }}$
Fig.7 shows some sample foreground and background color histograms. We can also observe that color histograms classify efficiently foreground regions. To confirm this observation, we have examined the distance between color histograms of background regions. The obtained results as we can observe on histograms (d, e, f); confirm that color histogram refines classification results obtained by texture histograms. However, these results may although be improved with edge histogram. As is shown in Fig.7, color histogram length equals to 400 bins. To further increase the characterization of ROI, we propose an additional edge ROI description. This edge description algorithm is combined with the proposed ROI color and texture description.

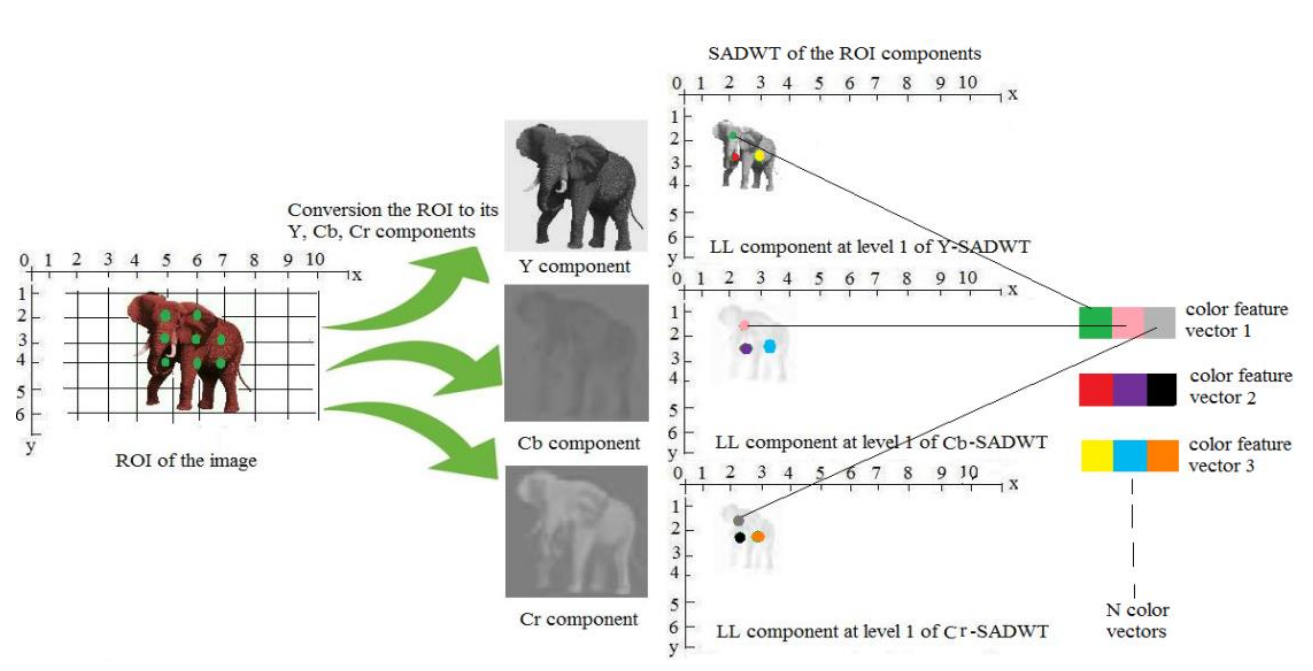

Fig.6. Color Features Construction Method using SA-DWT, Green Points on the Selected Region Represent an Arbitrary Chosen Set of Region Pixels. N Represents The Number of Wavelet Coefficients Contained in the LL Sub Band.

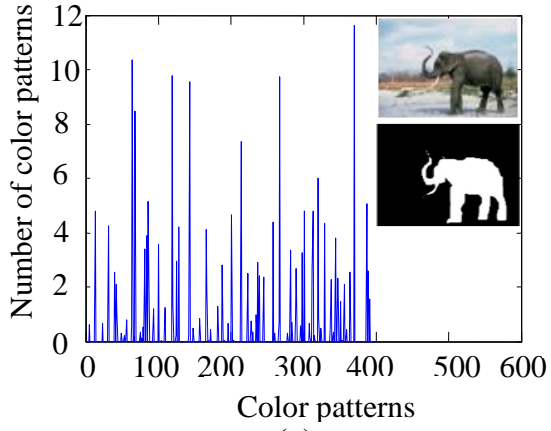

(a)

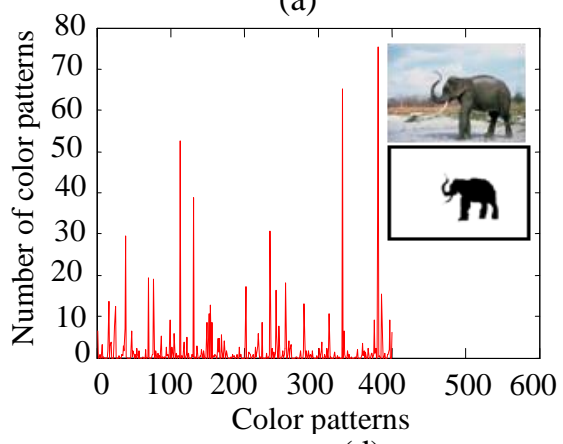

(d)

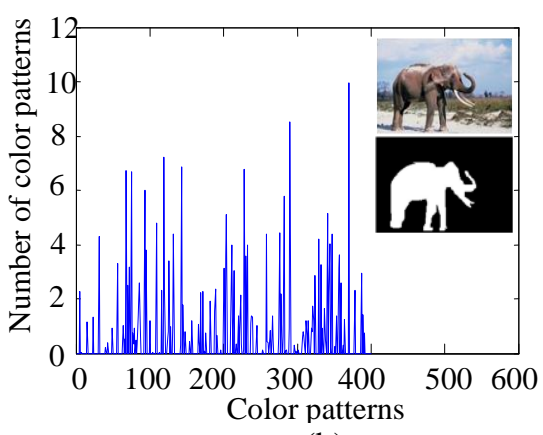

(b)

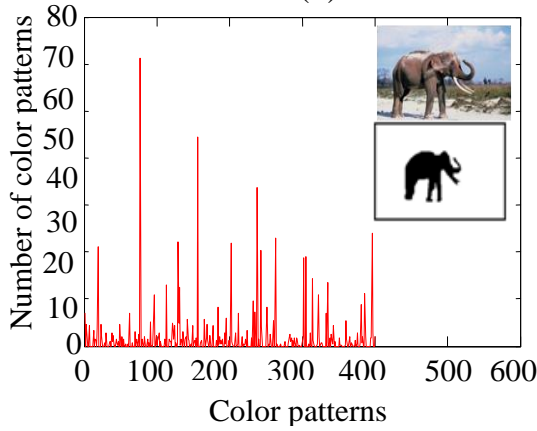

(e)

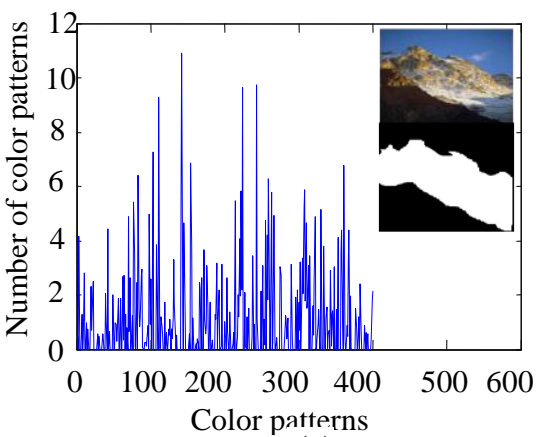

(c)

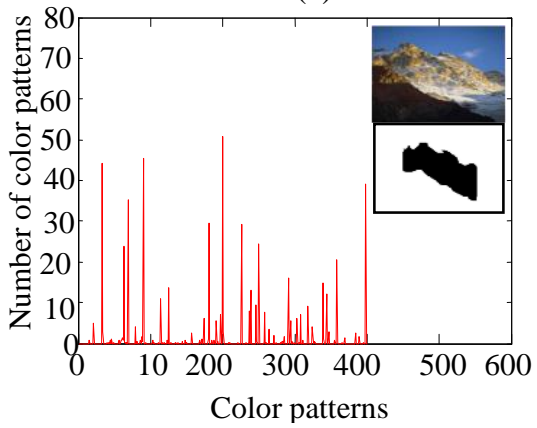

(f)

Fig.7. Examples of Color Histograms. (a), (b), (c) Color Histograms of Foreground Regions of two Elephant Images and one Mountain Image. (d), (e), (f) Color Histograms of Background Regions. 


\section{Edge descriptor construction}

Since the above texture and color features are not designed to give information about region edges, which is important to classify similar texture and color image regions, such sky background. An edge feature description is adopted in this paper. In this work the edges are used to describe the region boundary by its DLEP. The first step is to segment the approximation image of the component $\mathrm{Y}\left(w_{Y}^{L L}\right)$ into $3 * 3$ blocks. Edge characterization method is then applied on contour blocks. As shown in Fig.8, coefficients on the outside of the region are replaced with zeros.

In the following algorithm we explain our DLEP calculation method in $0^{\circ}$ direction.

Algorithm 4:

\begin{tabular}{l}
\hline Input: low-pass sub band wavelet transform. \\
\hline Step 1:Construct edge vectors as is shown in Fig.8. \\
Step 2: Construct centroid histogram $\left(\mathrm{His}_{\mathrm{center}}\right.$ ) for each class, \\
with V1_kmeans [26]. \\
Step 3: Construction of edge histograms in $0^{\circ}$ direction with \\
sparse algorithm. \\
\hline Output: $\mathrm{H}_{0}$ histograms.
\end{tabular}

This algorithm is then applied in the $45^{\circ}, 90^{\circ}$ and $135^{\circ}$ orientations. In Fig.9, edge histograms are created for 2 similar images of elephant and one image of mountain. Each edge histogram is the concatenation of edge histograms in: $0^{\circ}, 45^{\circ}, 90^{\circ}$ and $135^{\circ}$. Compared to color and texture histograms for the same images, we can observe that edge histogram compares efficiently texture orientations. For instance, histograms $(a, b, c)$ show that patterns number 7, 21, 23 and 45 (indexed by red elliptic form) appear only in elephant foregrounds, in this example we can observe that elephant backgrounds (sky) had also similar edge patterns distribution, which is different from sky edge patterns in the mountain image. For each edge histogram, we consider only first 100 bins. As stated before, using color and texture histograms for this example is not necessarily efficient for background (sky) characterization.

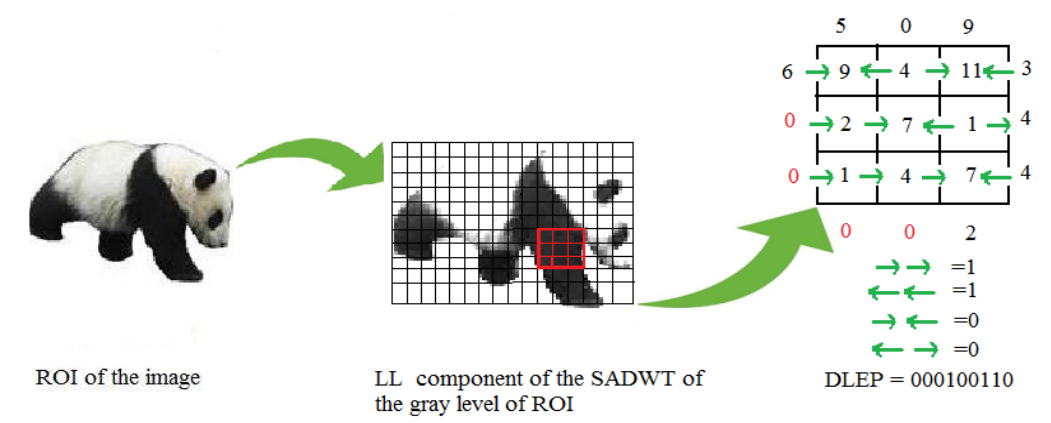

Fig. 8. edge construction of one $3 * 3$ blocks in $0^{\circ}$ direction, red block is chosen arbitrary.

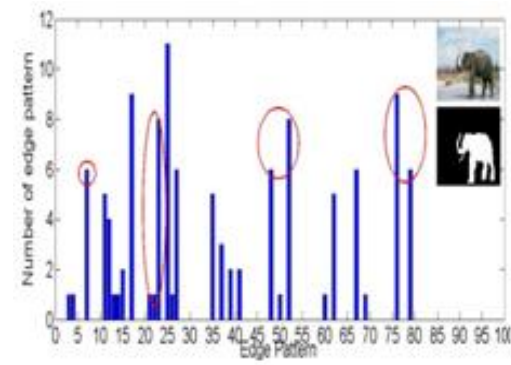

(a)

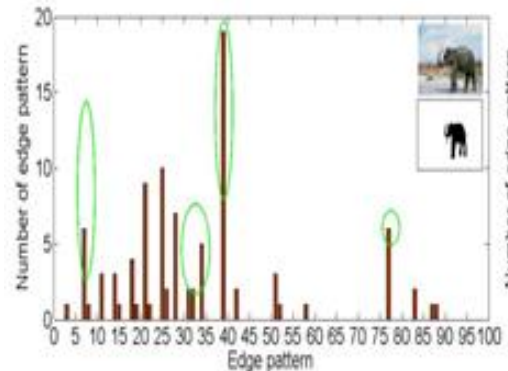

(d)

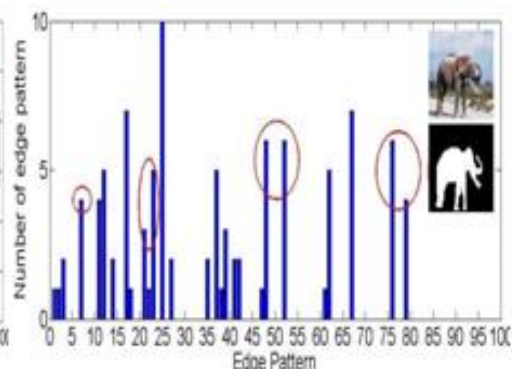

(b)

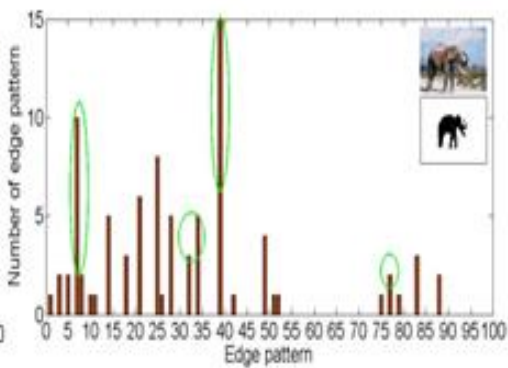

(e)

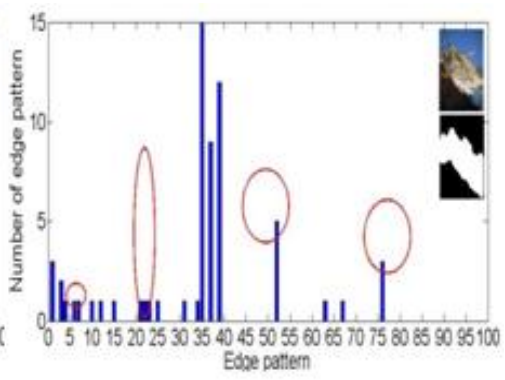

(c)

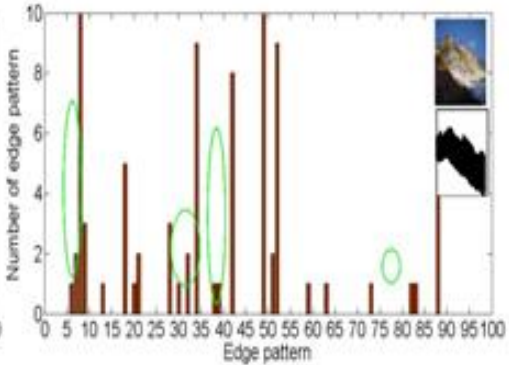

(f)

Fig.9. Examples of Edge Histograms. (a), (b), (c) Edge Histograms of Foreground Regions of two Elephant Images and one Mountain Image. (d), (e), (f) Edge Histograms of Background Regions. 
For this reason we use edge histograms, which are particularly discriminate for images backgrounds: common patterns are indicated by green elliptic form.

\section{IMAGE RETRIEVAL AND EXPERIMENTS}

In this part, we compare our method to some content and region based image retrieval schemes. Obtained results demonstrate the effectiveness of the proposed method. Our tests are achieved on two parts of Corel database (DB1, DB2) [28], this real-world images database consists of $256 * 384$ or $384 * 256$ RGB images, and it is organized into 10 images class: African, building, beach, dinosaurs, elephants, flowers, horses, mountains and food. With the same image background in different image categories: elephant, mountain, beach, and building, the challenge is how to eliminate the negative effect of common background on image classification process.

On other words, the state of art of RBIR methods shows that this common background such as sky for example, can produce poor image retrieval performances. We can also see that only the background edge feature can distinguish between images class. For this reason, we try to address this problem by using edge information as crucial classification feature. To analyze and evaluate the retrieval scheme performances, we use the precision and recall as statistical comparison parameters.

These parameters are defined by the following formulas:

Some reasons are due to the characteristics

$$
\begin{aligned}
& \text { Precision }=\frac{\text { Number of relevant images retrieved }}{\text { Total number of images retrieved }} \\
& \text { Recall }=\frac{\text { Number of relevant images retrieved }}{\text { Total number of relevant images in database }}
\end{aligned}
$$

Moreover, we use the average precision as an additional parameter of comparison.

The proposed image retrieval scheme can be summarized as follows:

1. The selected Corel images are firstly segmented into foreground and background regions. Each segmented image is then converted into $\mathrm{Y}, \mathrm{C}_{\mathrm{b}}, \mathrm{C}_{\mathrm{r}}$ color space.

2. Sub bands wavelet coefficients for each image are obtained according to the SA-DWT decomposition described in algorithm1.

3. Based on the obtained wavelet sub bands, we construct the color, texture and edge patterns, as described in algorithm 2, 3 and 4 respectively.

4. For each image, we get 3 histograms (edge, color and texture histogram):

The color histogram $H_{c}$ is the combination of the $H_{L L_{W}}$ histograms of the image regions

$$
H_{c}=\left[\omega_{p c} * H_{L L_{r 1}}\left(1-\omega_{p c}\right) * H_{L L_{r 2}}\right]
$$

$\omega_{p c}$ the color weight parameter.

The texture histogram $H_{T}$ is the combination of the regions texture histograms $\left(H_{T_{r}^{i}}\right)$.

Where $r$ represents image region.

As is mentioned above, for each texture characterization, we achieve three DWT level decomposition.

$$
H_{T}=\left[\omega_{p T} * H_{T_{r 1}} \quad\left(1-\omega_{p T}\right) * H_{T_{r 2}}\right]
$$

Where

$$
\begin{aligned}
& H_{T_{r 1}}=\left[H_{T_{r 1}^{1}} H_{T_{r 1}^{2}} H_{T_{r 1}^{3}}\right] \\
& H_{T_{r 2}}=\left[H_{T_{r 2}^{1}} H_{T_{r 2}^{2}} H_{T_{r 2}^{3}}\right]
\end{aligned}
$$

$\omega_{p T}$ the texture weight parameter.

The edge histogram $H_{E}$ is the combination of the $H_{0,45,90,135}$ histograms of the image regions

$$
H_{E}=\left[\omega_{p E} * H_{E_{r 1}}\left(1-\omega_{p E}\right) * H_{E_{r 2}}\right]
$$

Where

$$
\begin{aligned}
& H_{E_{r 1}}=\left[H_{0_{r 1}} H_{45_{r 1}} H_{90_{r 1}} H_{135_{r 1}}\right] \\
& H_{E_{r 2}}=\left[H_{0_{r 2}} H_{45_{r 2}} H_{90_{r 2}} H_{135_{r 2}}\right]
\end{aligned}
$$

$\omega_{p E}$ The edge weight parameter.

Weight parameters are used to outperform image matching performance.

The database contains different image classes, for each manipulation we consider each image as the query one $\left(I_{q}\right)$, and we compute the similarity between $I_{q}$ and all images database. The metric used to assess the similarity is defined by the $\chi^{2}$ distance as follows:

$$
d\left(H_{q}, H_{c}\right)=\sum_{i=1}^{L} \frac{\left(H_{q}(i)-H_{C}(i)\right)^{2}}{H_{q}(i)+H_{c}(i)}
$$

$H_{q}(i)$ and $H_{c}(i)$ denote the $\mathrm{i}^{\text {th }}$ bin value of the query or candidate histogram respectively.

$\mathrm{L}$ represents the number of bins or histogram length.

In order to combine edge, texture and color distances, we must normalize the distances before the combination step, by applying the following formula:

$$
d_{n o r}\left(H_{q}, H_{C}\right)=\frac{d\left(H_{q}, H_{C}\right)-\min \left(d\left(H_{q}, H_{C}\right)\right)}{\max \left(d\left(H_{q}, H_{C}\right)\right)-\min \left(d\left(H_{q}, H_{C}\right)\right)}
$$

min and max compute the maximum and minimum values of distances between different image histograms.

5. We finally, evaluate the proposed algorithm for different values of the weight parameters $\omega$ and $\alpha$ :

$d_{(q, c)}=\omega * d i s_{\text {nor }}^{c}+(1-\omega) *\left[\alpha^{*} d i s_{\text {nor }}^{T}+(1-\alpha)^{*} d i s_{\text {nor }}^{E}\right]$

Where: 


$$
\begin{gathered}
d i s_{\text {nor }}^{c}=d i s_{n o r}\left(H_{c q}, H_{c c}\right) \\
d i s_{\text {nor }}^{T}=d i s_{\text {nor }}\left(H_{T q}, H_{T c}\right) \\
d i s_{\text {nor }}^{E}=d i s_{\text {nor }}\left(H_{E q}, H_{E c}\right)
\end{gathered}
$$

\section{A. Experiments}

Most of existing wavelet based RBIR systems uses fixed windows sizes [29], or padding algorithm [9] to limit the region of interest. Compared to the original regions, these modified regions are more heterogeneous. The solution is to adapt the transformation to the original region exactly. Using SA-DWT can perform this task, and outperforms RBIR performances. To prove this, we achieve different tests. First, we conduct two experiences on database DB1 to define the optimal values of weight parameters; here we just show the experiment results obtained to define the weight parameters $\omega$ and $\alpha$.

As shown in Fig.10, we test our approach according to different values of the weight parameters $\omega$ and $\alpha$. We vary these parameters to evaluate the performance of retrieval, and to define the optimal values. $\omega_{\text {optimal }}=5$, $5, \alpha_{\text {optimal }}=4,5$ appear to be the optimal values. In fig.10, we can observe that better performances are obtained for small values of $\alpha$. Optimal weight parameters values are used for all experiments.

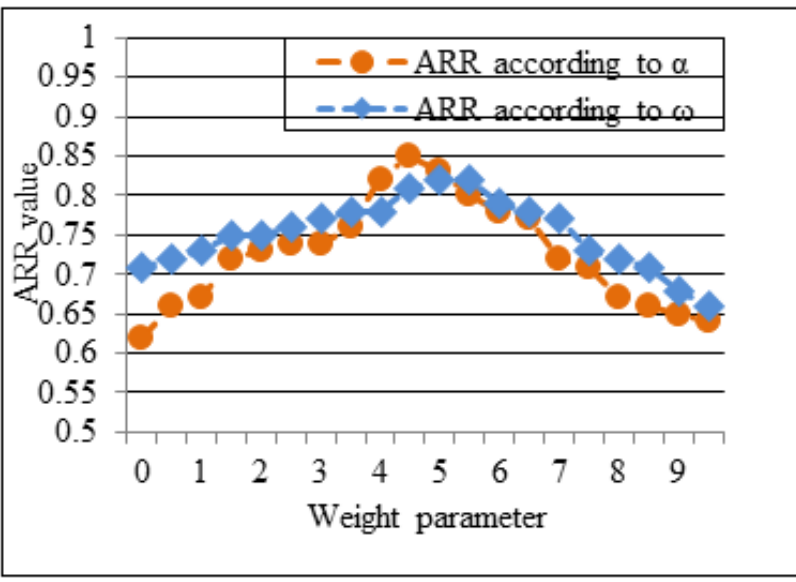

Fig.10. Definition of Optimal Weight Parameters.

\section{B. Comparison of the proposed approach with CBIR methods}

Table 1 shows the recall values obtained with our method and the following CBIR methods: to $\mathrm{K}$. Prasanthi method [14], Cong method [19]. DLEP method [22], Jhanwar et al method [30], HSV Hist method [31]. The number of returned images $n=100$. In table 2 , we present results in term of precision parameter. For each test, $\mathrm{n}=20$ represents the number of returned images. These tables confirm that the proposed method outperforms all proposed CBIR methods in term of average retrieval precision $82,47 \%$ compared to: $58,7 \%$ for Jhanwar et al, 67,7 achieved by HSV Hist, and to 63,7 for K. Prasanthi. Used Cong, and DLEP algorithms outperform these methods $(78,46 \%$ to $75,43 \%)$. It is clear that the proposed method outperforms all proposed CBIR methods in term of average retrieval recall and precision parameters.

\section{Comparison of the proposed approach with RBIR methods}

\section{Experiment \#1}

The method proposed in [29], introduces the edge information by using fixed $4 * 4$ windows to cluster the

\begin{tabular}{|c|c|c|c|c|c|c|}
\hline \multirow{2}{*}{ Category } & \multicolumn{6}{|c|}{ Recall $(n=100)(\%)$} \\
\hline & Jhanwar et al & HSV Hist & K. Prasanthi & DLEP & Cong et al & Proposed method \\
\hline Africans & 32.2 & 42.9 & 50.5 & 39.7 & 55.1 & 58.3 \\
\hline Beaches & 29.0 & 19.4 & 28.5 & 37.3 & 46.4 & 53.6 \\
\hline Buildings & 27.7 & 34.9 & 49.5 & 34.9 & 53.2 & 62.8 \\
\hline Buses & 48.6 & 63.7 & 74.8 & 74.1 & 78.0 & 83.3 \\
\hline Dinosaurs & 81.4 & 90.3 & 91.8 & 88.0 & 93.0 & 98.2 \\
\hline Elephants & 21.4 & 29.0 & 36.5 & 29.0 & 45.4 & 53.2 \\
\hline Flowers & 63.5 & 32.8 & 54.5 & 70.8 & 72.4 & 77.4 \\
\hline Horses & 35.8 & 50. & 55.1 & 41.7 & 58.1 & 65.3 \\
\hline Mountains & 21.7 & 14.5 & 22.2 & 29.0 & 39.5 & 52.1 \\
\hline Food & 29.0 & 47.8 & 51.7 & 47.0 & 53.6 & 53.2 \\
\hline Mean & 39.0 & 42.6 & 51.5 & 49.16 & 59.47 & 65.74 \\
\hline
\end{tabular}
image into a set of non overlapping blocks.

Table 1. Comparison of Recall Proposed Method with other CBIR Techniques. 
Table 2. Comparison of Precision Proposed Technique with other CBIR Techniques

\begin{tabular}{|c|c|c|c|c|c|c|}
\hline \multirow{2}{*}{ Category } & \multicolumn{6}{|c|}{ precision $(n=20)(\%)$} \\
\hline & Jhanwar et al & HSV Hist & K. Prasanthi & DLEP & Cong et al & Proposed method \\
\hline Africans & 53.15 & 57.2 & 66.7 & 74.3 & 76.3 & 80.1 \\
\hline Beaches & 43.85 & 53.8 & 32.2 & 65.6 & 63.2 & 75.3 \\
\hline Buildings & 48.7 & 63.5 & 56.8 & 75.4 & 73.1 & 77.1 \\
\hline Buses & 82.8 & 96.1 & 83.4 & 98.2 & 92.2 & 99.2 \\
\hline Dinosaurs & 95 & 100.1 & 99.8 & 99.1 & 95.3 & 99.5 \\
\hline Elephants & 34.85 & 40.1 & 50.6 & 63.3 & 64.1 & 67.8 \\
\hline Flowers & 88.35 & 90.3 & 66.2 & 94.1 & 95.2 & 96.0 \\
\hline Horses & 59.35 & 67.4 & 87.2 & 78.3 & 68.5 & 88.2 \\
\hline Mountains & 30.8 & 39.0 & 24.7 & 51.3 & 52.1 & 59.2 \\
\hline Food & 50.4 & 67.6 & 69.9 & 85.0 & 74.3 & 82.3 \\
\hline Mean & 58.7 & 67.7 & 63.7 & 78.46 & 75.43 & 82.47 \\
\hline
\end{tabular}

In this method the features like texture, color, and edge are extracted based on wavelet transform coefficients. From Fig.11, we can observe that:

- The background has brought positive influence on the dinosaur retrieval, because all images of the class dinosaur have the same color features.

- Poor results obtained by method explained in [29] on building, beach and elephant class. We can observe a significant retrieval improvement after using of the proposed method.

The extraction of texture information in $0^{\circ}, 45^{\circ}, 90^{\circ}$ and $135^{\circ}$ improve significantly the performance, compared to [29] method which extracts texture feature only in diagonal, vertical and horizontal directions.

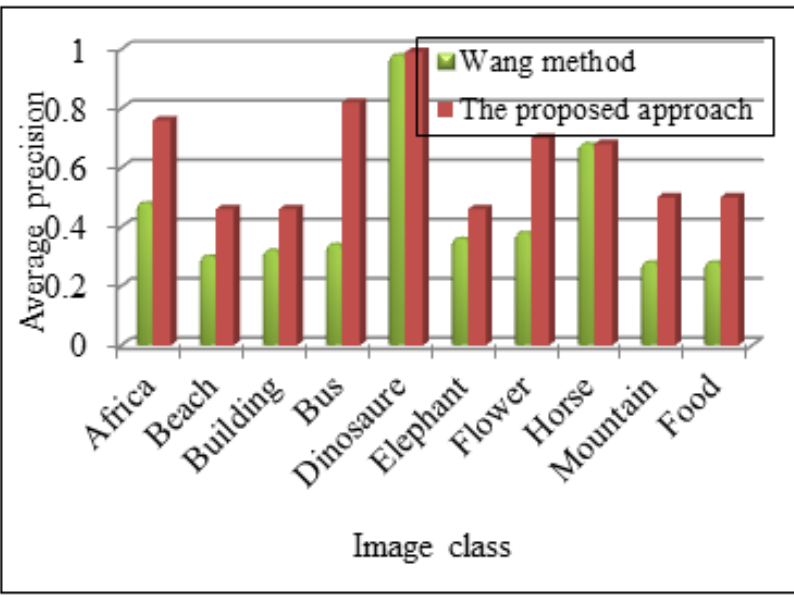

Fig.11 Comparison of the Proposed Method and Wang et al Method [9] Performances based on the Average Precision Measure.

\section{Experiment \#2}

As explained above, in their paper [18] Natsev et al propose a system based on separate windowing approach, to capture the shape information by the DWT transform. However, the windows used in this method can contain different regions or just a part of a given region. For this reason, our method is based on the mask information which limits the transformation to only the region of interest; we also use the edge histogram to improve our method performance. As shown in Fig.12 we can confirm that the proposed method is a real representative regions method.
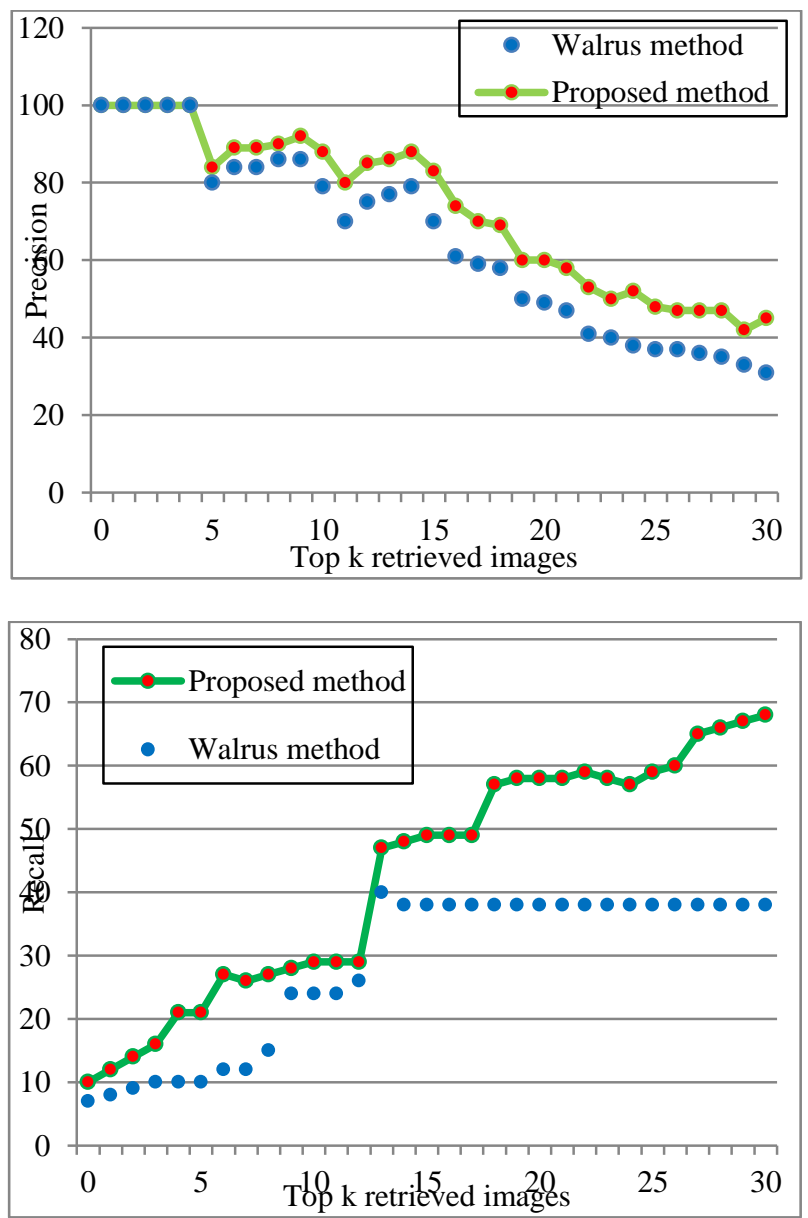

Fig.12. Comparison Recall, Precision Graphs of WALRUS and Proposed Method

In other words, this figure gives the compared results 
between WALRUS and our RBIR approach on Corel 1000 image database, and shows that combined histograms proposed method provides better retrieval performance, by matching and taking into account different regions features.

\section{Experiment \#3}

Curvelet based RBIR method [9] is a solution proposed by Md. Monirul Islam et al to overcome drawbacks of wavelet based RBIR methods. For this reason, we compare this method to our approach. In this section, we conduct an experiment on DB2 consisting of 5100 Corel images.

Fig.13 shows the improvement of our approach compared to curvelet based RBIR method. As explained in [9] each irregular region must be converted to a regular region, which produces significant change in information to be transformed due to the conversion irregular region to rectangular regions.

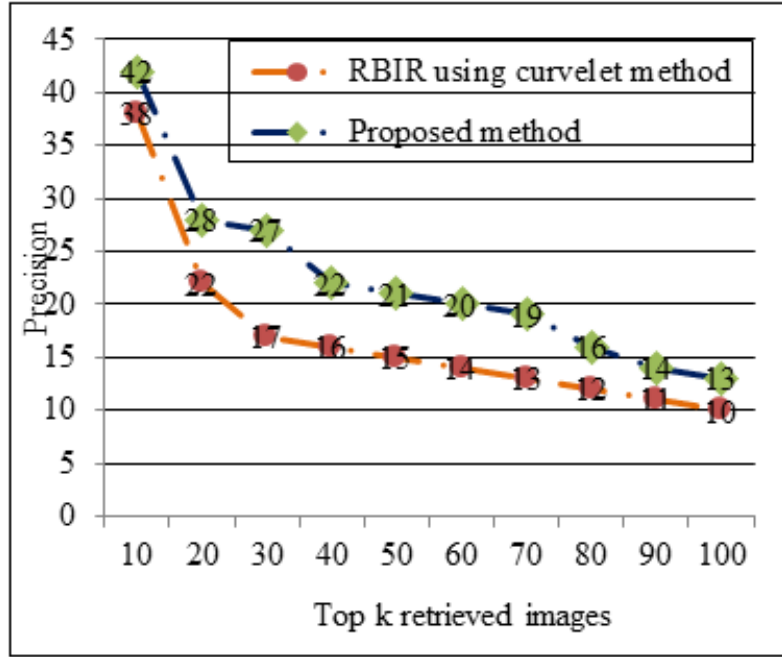

Fig.13. Comparison of Precision Graphs of Curvelet Based RBIR Method and Our Proposed Method

Our approach differs from related RBIR methods, by considering only the region to be transformed, without extrapolation operations. For this reason our method proves its ability to characterize efficiently image regions, and to preserve spatial and spectral region properties.

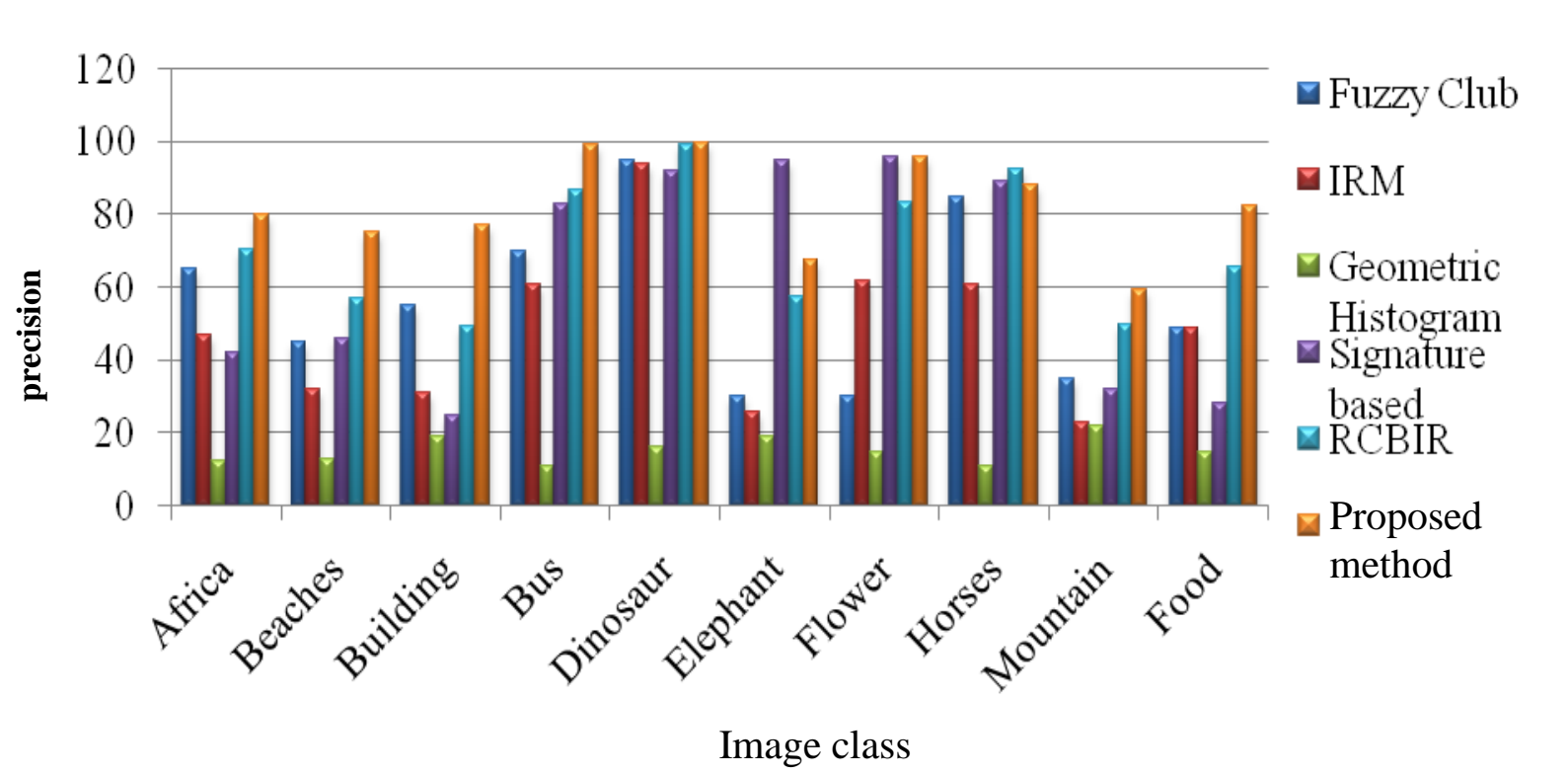

Fig.14. Comparison of Proposed Method (PM) with other RBIR Methods.

In Fig.14, we compare the proposed RBIR method with other RBIR algorithms (RCBIR method [12], Integrated Region Matching IRM [32], Fuzzy club method [33], Geometric histogram method [34], Signature based method [35]). We use the same approach for evaluation, as this proposed in [22], since we use the method proposed in [22], in the proposed method. For each images class, we randomly select 20 images as query. Returned images, for each query are used to compute the precision parameter.

Based on the top 20 images, we compute the average precision. As can be observed, the proposed method outperforms other all methods in all images classes, excepted elephant, flowers, and horses images classes. The best image retrieval performances are recorded in bus, dinosaurs' images classes.

\section{CONCLUSION AND FUTURE WORK}

The objectives of this work is to improve the retrieval performances of existing object image retrieval methods, and solve the problem of edge description by wavelet transform. Based on an adaptive region transformation 
scheme, the proposed construction histogram method is based on combination regions histograms, to construct three histograms for each image in the database.

Tested on DB1 and DB2 databases, our method has demonstrated more efficiency then other RBIR methods. Further our RBIR scheme can be extended to 3D image retrieval, by applying the proposed method on $2 \mathrm{D}$ views constructing the 3D object (light field). This allows improving previous obtained results on light field, which is proposed in [2].

\section{ACKNOWLEDGMENT}

The work is currently supported by the Partenariat Hubert Curien PHCTASSILI. Under grant No 12MDU864. The authors thank for their financial supports.

\section{REFERENCES}

[1] K. Belloulata, L. Belhallouche, A. Belalia, K. Kpalma, "Region based image retrieval using shape-adaptive DCT”, China SIP, pp. 470-474, July 2014.

[2] L. Belallouche, K. Belloulata, K. Kpalma. "Light field retrieval in compressed domain", $21^{\text {st }}$ international conference in central Europe on computer graphics, visualization and computer vision, pp. 45-48. 2013.

[3] H. Abrishami, M. T. T. Khajoie, A. H. Rouhi, M. S. Tarzjan, "Wavelet correlogram: A new approach for image indexing and retrieval Pattern Recognition", Pattern Recognition journal, vol 38, pp. 2506 - 2518. 2005.

[4] W. Y. Gao, K. Chan. "A Review of Region-Based Image Retrieval". Journal Signal Processing System, pp. 59:143-161. 2010.

[5] Tsai, C.-F., McGarry, K., \& Tait, J. "Image classification using hybrid neural network". In Proceedings of the ACM SIGIR conference on research and development in information retrieval, pp. 431-432, 2003.

[6] E R Vimina1 and K Poulose Jacob, "A Sub-block Based Image Retrieval Using Modified Integrated Region Matching", International Journal of Computer Science Issues, Vol.10, Issue 1,No 2, pp. 686-692, 2013.

[7] D. G. Lowe, "Object recognition from local scaleinvariant features", international conference on computer vision, pp.1150-1157, 1999.

[8] J. Yu, Z. Qin, T. Wan, X. Zhang, "Feature integration analysis of bag-of-features model for image retrieval", Neuro computing journal, pp. 355-364, 2013.

[9] D. Zhang, Md. Monirul Islam, G. Lu, I. J. Sumana, "rotation invariant curvelet features for region based image retrieval", International Journal of Computer Vision , pp. 187-201, 2012.

[10] K. Prasanthi Jasmine, P. Rajesh Kumar, "color and rotated M-Band dual tree complex wavelet transform features for image retrieval", international journal of image, graphics and signal processing, pp. 1-10, 2014.

[11] C-Y. Wang, X. Zhang, R. Shan, X. Zhou, "grading image retrieval based on DCT and DWT compressed domains using Low-Level features", Journal of Communications, pp. 64-73, 2015.

[12] S. I. Ibrahim, A. Abuhaiba, A. Ruba, A.Salamah, "efficient global and region content based image retrieval", international journal of image, graphics and signal processing, pp. 38-46, 2012.

[13] M. Li, R. C. Staunton, "optimum Gabor filter design and local binary patterns for texture segmentation", journal pattern recognition, pp. 664-672, 2008.

[14] K. Prasanthi Jasmine, P. Rajesh Kumar, "color and local maximum edge patterns histogram for content based image retrieval", international journal of intelligent systems and applications, pp. 66-74, 2014.

[15] Y. Chen, J. Wang, "A region-based fuzzy feature matching approach to content-based image retrieval", IEEE transactions on pattern analysis and machine intelligence, pp. 1252-1267, 2002.

[16] C. Huang, Y. Han, Y. Zhang, "A method for object-based color image retrieval", $9^{\text {th }}$ international conference on fuzzy systems and knowledge discovery, pp. 1659 - 1663, 2012.

[17] Y. Sun, S. Ozawa, "HIRBIR: A hierarchical approach to region-based image retrieval", multimedia systems journal, pp. 559-569, 2005.

[18] A. Natsev, R. Rastogi, K. Shim, "WALRUS: A similarity retrieval algorithm for image databases", IEEE transactions on knowledge and data engineering, pp. 301 $-316,2004$.

[19] C. Bai, J Zhang, Z Liu, W-L Zhao, "K-means based histogram using multiresolution feature vectors for color texture database retrieval", Journal multimedia tools and applications, pp. 1469-1488, 2015.

[20] S. Li, W. Li, "shape adaptive discrete wavelet transforms for arbitrarily shaped visual object coding", IEEE transactions on circuits and systems for video technology, pp. $725-743,2000$.

[21] C-T. Huang, P-C. Tseng, L-G. Chen, "VLSI architecture for lifting-based shape-adaptive discrete wavelet Transform with Odd-symmetric filters", Journal of VLSI signal processing, pp. 175-188, 2005.

[22] S. Murala, R. P. Maheshwari, R. Balasubramanian, "Directional local extrema patterns: a new descriptor for content based image retrieval", International journal of multimedia information retrieval, pp. 191-203, 2012.

[23] D Gabor, "Theory of communication. Part 1: The analysis of information", Journal of the institution of electrical engineers, -Part III. - IET, pp. 429-441, 1946.

[24] K. Seetharaman, M. Kamarasan, "Statistical framework for content-based medical image retrieval based on wavelet orthogonal polynomial model with multiresolution structure", international journal multimedia information retrieval, pp. 53-66, 2014.

[25] S. Mallat, "A theory for multiresolution signal decomposition: The wavelet representation", IEEE transactions on pattern analysis and machine intelligence, pp. 674-693, 1989.

[26] http://www.vlfeat.org/matlab/vl_kmeans.html.2014.

[27] http://spams-devel.gforge.inria.fr/.2014.

[28] https://sites.google.com/site/dctresearch/Home/content based-image-retrieval.2014.

[29] J.Z. Wang, J. Li, G. Wiederhold, "Simplicity Semanticssensitive integrated matching for Picture librairies", IEEE Transactions on pattern analysis and machine intelligence, pp. 1- 17, 2001.

[30] N. Jhanwara, S. Chaudhuri, G. Seetharamanc, and B. Zavidovique, "Content based image retrieval using motif co-occurrence matrix", Image and Vision Computing, pp. 1211-1220. 2004

[31] Subrahmanyam Murala, R. P. Maheshwari, R. Balasubramanian, "Expert content-based image retrieval system using robust local patterns", J. Visual communication and image representation, pp. 1324-1334, 2014.

[32] J. Li, J. Wang, and G. Wiederhold, "Integrated region 
matching for image retrieval," in Proc. 2000 ACM Multimedia Conf., Los Angeles, pp. 147-156, 2000.

[33] R. Zhang and Z. Zhang, "A clustering based approach to efficient image retrieval," in Proc. 14th IEEE Int. Conf. Tools with Artificial Intell. (ICTAI'02), Washington, DC, pp. 339-346, 2002.

[34] A. Rao, R. Srihari, and Z. Zhang, "Geometric histogram: A distribution of geometric configuration of color subsets," in Proc. SPIE Conf. Electronic Imaging 2000, vol. 3964-09, San Jose, CA, pp. 91-101, 2000.

[35] D. Lakshmi, A. Damodaram, M. Sreenivasa, and J. Lal, "Content based image retrieval using signature based similarity search,” Indian J. Science and Technology, vol. 1, no. 5, pp. 80-92, Oct. 2008.

[36] Y. Liu, X. Zhou, W-Y. Ma, "Extracting texture features from arbitrary-shaped regions for image retrieval", Multimedia and Expo, ICME, IEEE International Conference, pp. 1891 - 1894, 2004.

[37] Y. Liu, D. Zhang, G. Lu, W-Y. Ma, "Study on texture feature extraction in region-based image retrieval system", Multi-Media Modelling Conference, pp. 264-271, 2006.

\section{Authors' Profiles}

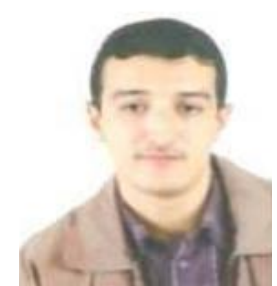

Lakhdar BELHALLOUCHE, was born in Algeria 1981. He received his master degree in image processing and vision from Djillali Liabbes University, Algeria in 2008. Currently, he prepares his Ph.D in electronics in Djillali Liabbes University. His major fields of interest are Image Retrieval, Digital image Processing and Pattern Recognition.

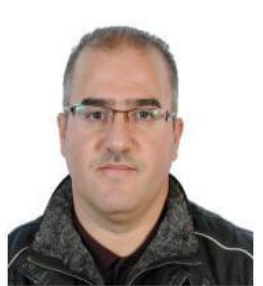

Kamel BELLOULATA was born in Algeria in 1969. He received the D.E.A. and $\mathrm{Ph} . \mathrm{D}$. degrees in signal, image, and speech processing from the Institut National des Sciences Appliquées (INSA) de Lyon, France, in 1994 and 1998, respectively. From September 1998 to august 1999, he was a Postdoctoral Fellow at INRS-Télécommunications, Montréal, Qc, Canada. From September 1999 to December 2001, he has been an
Assistant Professor with the Department of Electrical Engineering of University of Moncton, NB, Canada. Since January 2002, he has been an Associate Professor with the Electrical and Computer Engineering Department of University of Sherbrooke, Qc, Canada. Currently he is Full Professor at the University of Sidi Bel Abbs, Algeria. His current research interests are in the areas of image and video compression, pattern recognition and semantic segmentation.

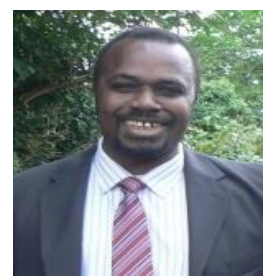

Kidiyo KPALMA is born in 1962 in Togo. After his secondary school, he joined the University of Rennes 1 in France from whom he obtained his M.Sc in Signal Processing in 1988. He then joined the National Institute for Applied Sciences of Rennes (INSA) for his PhD studies in Image Processing: he received this degree in 1992 and then became Associate Professor at INSA, in 1994, where he teaches Signal and Systems, Signal Processing and DSP. In 2009 he received his HDR (Habilitation à diriger des recherches) degree in Signal Processing and Telecommunications from the University of Rennes 1 and then became Professor since 2014 at INSA. As a member of the Department of Image and Automatic of the Institute of Electronics and Telecommunications of Rennes (IETR), his research interests are image analysis, pattern recognition, image segmentation, semantic segmentation, image fusion and remote sensing.

How to cite this paper: Lakhdar BELHALLOUCHE, Kamel BELLOULATA, Kidiyo KPALMA,"A New Approach to Region Based Image Retrieval using Shape Adaptive Discrete Wavelet Transform", International Journal of Image, Graphics and Signal Processing(IJIGSP), Vol.8, No.1, pp.1-14, 2016.DOI: 10.5815/ijigsp.2016.01.01 